\title{
Cordycepin induces apoptosis and autophagy in human neuroblastoma SK-N-SH and BE(2)-M17 cells
}

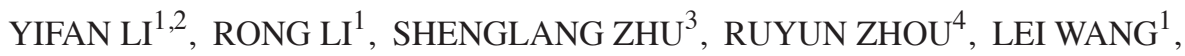 \\ JIHUI DU ${ }^{1}$, YONG WANG ${ }^{5}$, BEI ZHOU $^{1}$ and LIWEN MAI ${ }^{1}$ \\ ${ }^{1}$ Central Laboratory; ${ }^{2}$ Shenzhen Key Lab of Endogenous Infection; Departments of ${ }^{3}$ Nephrology, \\ ${ }^{4}$ Chinese Traditional Medicine Rheumatology and ${ }^{5}$ Gastroenterology, Affiliated Nanshan Hospital, \\ Guangdong Medical College, Shenzhen, Guangdong 518052, P.R. China
}

Received June 30, 2014; Accepted February 11, 2015

DOI: $10.3892 / \mathrm{ol} .2015 .3066$

\begin{abstract}
Cordycepin, also termed 3'-deoxyadenosine, is a derivative of the nucleoside adenosine that represents a potential novel class of anticancer drugs targeting the 3' untranslated region of RNAs. Cordycepin has been reported to induce apoptosis in certain cancer cell lines, but the effects of cordycepin on human neuroblastoma cells have not been studied. In the present study, an MTT assay revealed that cordycepin inhibits the viability of neuroblastoma SK-N-SH and BE(2)-M17 cells in a dose-dependent manner. In addition, cordycepin increases the early-apoptotic cell population of SK-N-SH cells, as determined by fluorescence-activated cell sorting analysis. The induction of apoptosis in neuroblastoma cells by cordycepin was further confirmed by western blotting, which revealed cleavage of caspase-3 and poly(adenosine diphosphate-ribose) polymerase 1 in the SK-N-SH and BE(2)-M17 cells. Cordycepin also induced the formation of a punctate pattern of light-chain 3 (LC3)-associated green fluorescence in the SK-N-SH cells transfected with a pEGFP-LC3 vector. Furthermore, western blotting revealed cleavage of LC3 A/B in cordycepin-treated neuroblastoma SK-N-SH cells. Taken together, the results indicate that cordycepin significantly increases apoptosis and autophagy in neuroblastoma cells, and may therefore be a drug candidate for neuroblastoma therapy, but requires additional evaluation.
\end{abstract}

\section{Introduction}

Neuroblastoma is the most common extracranial solid cancer in childhood and the most common cancer in infancy, arising from the developing sympathetic nervous system (1-3).

Correspondence to: Dr Yifan Li, Central Laboratory, Affiliated Nanshan Hospital, Guangdong Medical College, 89 Taoyuan Road, Shenzhen, Guangdong 518052, P.R. China

E-mail: liyifan2005@yahoo.com

Key words: cordycepin, apoptosis, autophagy, neuroblastoma
The prevalence of neuroblastoma is $\sim 1$ case in 7,000 live births (1-3). Approximately $40 \%$ of neuroblastoma patients present with a localized tumor, which appears as a large lump in the abdomen, pelvis, chest or neck, that may press on other parts of the body (4). The disease exhibits extreme heterogeneity, and is stratified into three risk categories, low, intermediate and high risk (3). These risk catergories are based on clinical and biological features, including MYCN copy number, histopathology, tumor ploidy in infants, patient age and tumor stage [according to the International Neuroblastoma Staging System (5)] (3). The risk categories are used to predict how likely it is that a child can be cured (6) and to stratify patients in clinical trials to determine the appropriate type and intensity of therapy (5). A higher-risk group indicates that there is less chance of a cure, and thus more intensive treatments are often required (5). Approximately $50 \%$ of patients exhibit metastasis to the lymph nodes, bone, bone marrow and liver, presenting with syndromes including bone pain and bone marrow failure (4). Occasionally, patients may exhibit renin-mediated hypertension (4). Notably, metastasis of neuroblastoma occurs prior to the appearance of any symptoms in 50-60\% of all neuroblastoma cases (7). A rare type of neuroblastoma, termed stage $4 \mathrm{~S}$, that accounts for $5 \%$ of neuroblastoma cases, presents as small localized primary tumors with metastases to the liver, skin or bone marrow, which usually regresses spontaneously (8). The majority of cases of stage 4S neuroblastoma are diagnosed in children aged $<1$ year old $(5,9)$. Certain neuroblastoma patients have an excellent prognosis, such as those with stage $4 \mathrm{~S}$ neuroblastoma (10), however, older children usually exhibit metastatic disease and thus have a worse prognosis. The overall five-year survival rate for neuroblastoma patients is 59\%, ranging from $47 \%$ (Eastern populations) to $67 \%$ (Western populations) (2). The majority of neuroblastomas are treated using conventional approaches, including surgery, radiation and chemotherapy (3). Although the success rate of current therapies for the treatment of patients with low- and intermediate-risk neuroblastoma is $>90$ and $70-90 \%$, respectively, only $\sim 30 \%$ of high-risk neuroblastoma patients have been successfully treated in the previous two decades (11-13). Therefore, research into novel agents that are able to more effectively treat neuroblastoma 
is necessary. At present, therapeutic approaches that exhibit less toxicity and increased specificity are being investigated. For example, 13-cis retinoic acid, which induces differentiation of neuroblastoma cells, has become a standard treatment for the management of high-risk neuroblastoma patients following marrow or stem cell transplantation (14). Other potential treatment modalities for neuroblastoma include drugs that target apoptosis or inhibit angiogenesis, targeted radiation therapy $(3,4)$ and immunotherapy $(12)$.

Cordycepin, also termed 3'-deoxyadenosine, originates from Ophiocordyceps sinensis, which is commonly used in Traditional Chinese Medicine (15). Cordycepin is an adenosine analogue that differs from adenosine by the deletion of oxygen in the 3 ' position of the ribose region (16). Therefore, certain enzymes are unable to discriminate between the two. Cordycepin may be phosphorylated to mono-, di- and triphosphate forms within cells, and participates in a number of biochemical reactions (16). For example, triphosphate cordycepin may be incorporated into RNA molecules, leading to the premature termination of RNA synthesis and inhibition of transcription elongation (17). Cordycepin is an example of a proposed new class of anticancer drugs that target RNA synthesis (18).

Previously, cordycepin has been revealed to induce apoptosis in a number of cancer cell lines, including breast (19), colon (20) and oral cancers (21), mouse leydig tumor (22), and oral squamous cell carcinoma (23). Cordycepin also induces autophagy in breast cancer cells (19) and has been used in a phase I study in combination with pentostatin to treat refractory acute lymphocytic or chronic myelogenous leukemia (Study ID, CDR0000065572) (24). However, the effects of cordycepin on neuroblastoma cells have not yet been examined.

The present study investigates the apoptotic and autophagic effects of cordycepin on the human neuroblastoma cell lines SK-N-SH and BE(2)-M17, in order to assess its feasibility as a drug candidate for neuroblastoma therapy.

\section{Materials and methods}

Chemicals. Hirsutella sinensis extract (0.199 g/ml), which was produced by fermentation and extraction using ethanol, water and enzyme digestion methods by Huadong Medicine (Hangzhou, Zhejiang, China), was provided by Dr Shenglang Zhu at Nanshan Hospital (Shenzhen, Guangdong, China). Hirsutella sinensis is the anamorphic, mycelial form of Cordyceps sinensis $(25,26)$. Cordycepin (catalog no., C3394) and 3-methyladenine (catalog no., M9281) were purchased from Sigma-Aldrich (St. Louis, MO, USA).

Cell lines and cell culture. The human neuroblastoma cell line SK-N-SH was obtained from the Cell Bank of Type Culture Collection of Chinese Academy of Sciences (Word Federation for Culture Collection registered no., 793; Shanghai, China). The human neuroblastoma cell line BE(2)-M17 was from the Cell Resource Center (Institute of Basic Medical Sciences, Chinese Academy of Medical Sciences \& Peking Union Medical College, Beijing, China). The cells were cultured using standard methods (27) in Dulbecco's modified Eagle's medium (DMEM; Hyclone, Logan, UT, USA) supplemented with $10 \%$ fetal bovine serum (FBS; Gibco, Carlsbad, CA, USA), $2 \mathrm{mmol} / \mathrm{l}$ L-glutamine (Sigma-Aldrich), 100 units/ml penicillin and $100 \mu \mathrm{g} / \mathrm{ml}$ streptomycin (Gibco), at $37^{\circ} \mathrm{C}$ in a $5 \% \mathrm{CO}_{2}$ atmosphere.

Vectors and transfection. The pEGFP-LC3 plasmid (plasmid no., 21073; Addgene, Inc., Cambridge, MA, USA) is a pEGFP-C1 plasmid (Clontech Laboratories, Inc., Mountainview, CA, USA) inserted with a 738-bp long cDNA sequence from rat microtubule-associated protein 1 light chain 3 (LC3). This sequence encodes a fusion protein composed of green fluorescent protein (GFP) at the N-terminus and LC3 at the C-terminus (28). In total, $1 \times 10^{6} \mathrm{SK}-\mathrm{N}-\mathrm{SH}$ cells/well were seeded into six-well plates, $24 \mathrm{~h}$ prior to transfection. The SK-N-SH cells were transfected with pEGFP-LC3 using FuGENE HD Transfection Reagent (Promega Corporation, Madison, WI, USA) and left for $48 \mathrm{~h}$, followed by selection with $800 \mu \mathrm{g} / \mathrm{ml} \mathrm{G} 418$ (Amresco, Inc., Framingham, MA, USA) for one week.

Cell morphology study. The SK-N-SH or BE(2)-M17 cells were seeded at a density of $1 \times 10^{6}$ cells/well into six-well plates $24 \mathrm{~h}$ prior to treatment. SK-N-SH cells were treated with various concentrations of Cordyceps sinensis extract. The SK-N-SH and BE(2)-M17 cells were treated with various concentrations of cordycepin. The morphology of each cell line was analyzed using an Axiovert 40 microscope (Zeiss, Oberkochen, Germany).

MTT assay. The SK-N-SH or BE(2)-M17 cells were seeded at a density of $10^{4}$ cells/well in 96 -well plates, $24 \mathrm{~h}$ prior to treatment. At $48 \mathrm{~h}$ subsequent to cordycepin treatment, the medium was removed and $100 \mu \mathrm{l}$ of serum free DMEM containing $0.5 \mathrm{mg} / \mathrm{ml}$ MTT (Sigma-Aldrich) was added to each well. The cells were then incubated at $37^{\circ} \mathrm{C}$ for $4 \mathrm{~h}$. Subsequent to the removal of the MTT media, $100 \mu 1$ dimethyl sulfoxide was added to each well and incubated at $37^{\circ} \mathrm{C}$ for $20 \mathrm{~min}$. The plate was incubated at room temperature for an additional 5 min with agitation, and the absorbance of the solution was subsequently quantified at a wavelength of $570 \mathrm{~nm}$, which was normalized against a reference value measured at a wavelength of $690 \mathrm{~nm}$ to account for debris, using the PARADIGM Detection Platform (Beckman Coulter, Brea, CA, USA).

Fluorescence-activated cell sorting (FACS). The SK-N-SH cells were cultured in DMEM supplemented with $10 \% \mathrm{FBS}$, $2 \mathrm{mmol} / 1 \mathrm{~L}$-glutamine, $100 \mathrm{units} / \mathrm{ml}$ penicillin and $100 \mu \mathrm{g} / \mathrm{ml}$ streptomycin at $37^{\circ} \mathrm{C}$ in a $5 \% \mathrm{CO}_{2}$ atmosphere. The SK-N-SH cells $\left(5 \times 10^{5}\right)$ were seeded into each well of a six-well plate and grown overnight, which was followed by treatment with $0,50,100$ or $200 \mu \mathrm{mol} / 1$ cordycepin for $48 \mathrm{~h}$. For the experiment measuring the effect of 3-methyladenine on cordycepin-induced apoptosis, SK-N-SH cells were treated with 0 or $150 \mu \mathrm{mol} / 1$ cordycepin, $5 \mathrm{mmol} / 13$-methyladenine, or combination of the latter two, for $48 \mathrm{~h}$. Untreated cells were used as the negative control. The free-floating and attached SK-N-SH cells from each well were harvested and pooled. The cells were washed with phosphate buffered saline (PBS) and stained using a Dead Cell Apoptosis kit with Annexin V Alexa Fluor 488 and propidium iodide (PI) (Invitrogen Life Technologies, Carlsbad, CA, USA), according to the 

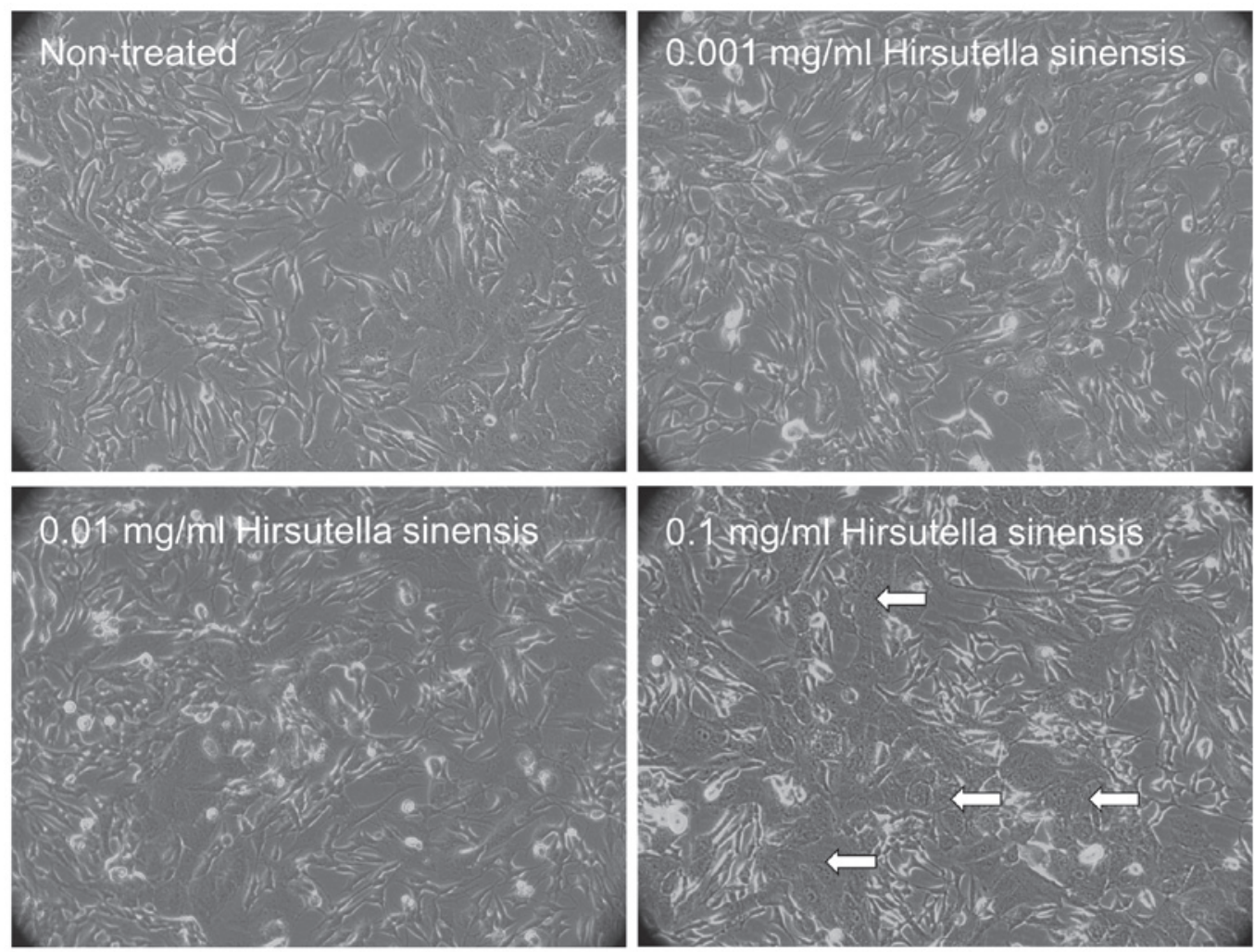

Figure 1. Morphology of SK-N-SH cells treated with Hirsutella sinensis extract. The SK-N-SH cells were treated with 0 , $0.001,0.01$ or 0.1 mg/ml Hirsutella sinensis extract for $72 \mathrm{~h}$. The white arrows indicate the phenotypical changes in the SK-N-SH cells that were observed under a phase-contrast microscope (magnification, $\mathrm{x} 20$ ).

manufacturer's instructions. The stained cells were analyzed using a Cytomics FC 500 flow cytometer (Beckman Coulter).

Westernblotanalysis. The antibodiesagainstcaspase-3(catalog no., 9665S), poly(adenosine diphosphate-ribose) polymerase 1 (PARP1; catalog no., 9542S) and LC3 A/B (catalog no., 4108S) were purchased from Cell Signaling Technology, Inc. (Danvers, MA, USA). The anti- $\beta$-actin antibody (catalog no., sc-47778) was purchased from Santa Cruz Biotechnology, Inc. (Dallas, TX, USA), and the whole-molecule anti-rabbit immunoglobulin (Ig)G-peroxidase (cat. no. A9169) and whole-molecule anti-mouse IgG-peroxidase antibodies (cat. no. A9044) were purchased from Sigma-Aldrich.

In total, $1 \times 10^{6}$ cells were lysed in $150 \mu 1$ cell lysis buffer (Cell Signaling Technology) containing an EDTA-free protease inhibitor cocktail (Roche Diagnostics, Basel, Switzerland). The protein concentrations were determined using a detergent-compatible protein assay kit (Bio-Rad Laboratories, Inc., Hercules, CA, USA). For each well, $30 \mu \mathrm{g}$ of protein lysate was combined with $2 \mu 12 \mathrm{X}$ sample buffer, comprising final concentrations of $60 \mathrm{mmol} / \mathrm{l}$ Tris $(\mathrm{pH}, 6.8)$, $10 \%$ glycerol, $2 \%$ sodium dodecyl sulfate and $5 \%$ mercaptoethanol. The lysate was then incubated at $100^{\circ} \mathrm{C}$ for $5 \mathrm{~min}$ to denature the proteins. The protein samples were loaded for electrophoresis, and then transferred to Immobilon-P membranes (Millipore, Billerica, MA, USA) using wet transfer apparatus. The membrane was blocked in $1 \%$ bovine serum albumin (Sigma-Aldrich) in PBS for $2 \mathrm{~h}$ at room temperature. The membranes were subsequently probed with primary antibodies overnight at $4^{\circ} \mathrm{C}$, followed by secondary antibody incubation at room temperature for $1.5 \mathrm{~h}$. The protein bands were visualized with enhanced chemiluminescence prime western blotting detection reagent (Amersham, GE Healthcare, Shanghai, China) and images were captured using the MiniChemi professional machine (SageCreation Science, Co., Ltd., Beijing, China).

Statistical analysis. Student's $t$-test was used to compare the viability of SK-N-SH and BE(2)-M17 cells measured by MTT assay. Statistical analyses were performed using Microsoft Excel 2010 software (Microsoft, Redmond, WA, USA). The results are presented as the mean \pm standard error of the mean. All P-values were two-tailed and $\mathrm{P}<0.05$ was considered to indicate a statistically significant difference.

\section{Results}

Morphological changes in neuroblastoma cells treated with Hirsutella sinensis extract. To examine the effects of Hirsutella sinensis extract on neuroblastoma cells, various concentrations of the extract, comprising $0,0.001,0.01$ and $0.1 \mathrm{mg} / \mathrm{ml}$, were incubated with SK-N-SH cells for $48 \mathrm{~h}$. Administration of $0.1 \mathrm{mg} / \mathrm{ml}$ Hirsutella sinensis extract markedly altered the cell phenotype, as indicated by an increased proportion of SK-N-SH cells possessing cell granulations that were visible under a phase-contrast microscope, compared with the untreated control (Fig. 1). Administration of 0.001 and $0.01 \mathrm{mg} / \mathrm{ml}$ Hirsutella sinensis extract resulted in no significant effect on the phenotype of the SK-N-SH cells (Fig. 1). 


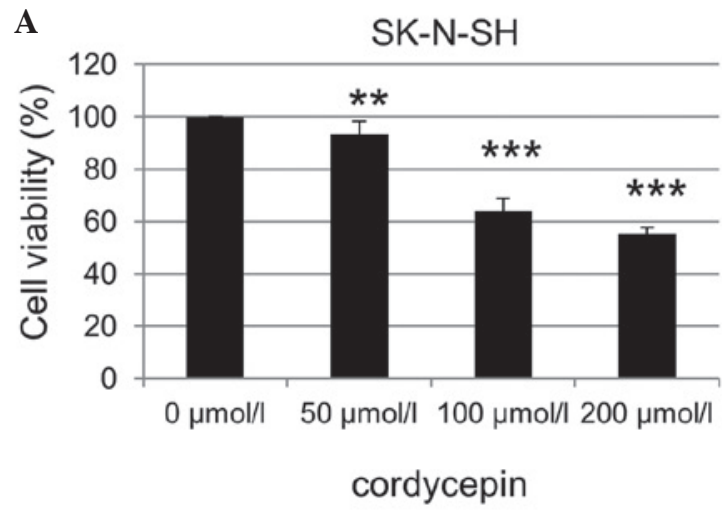

B
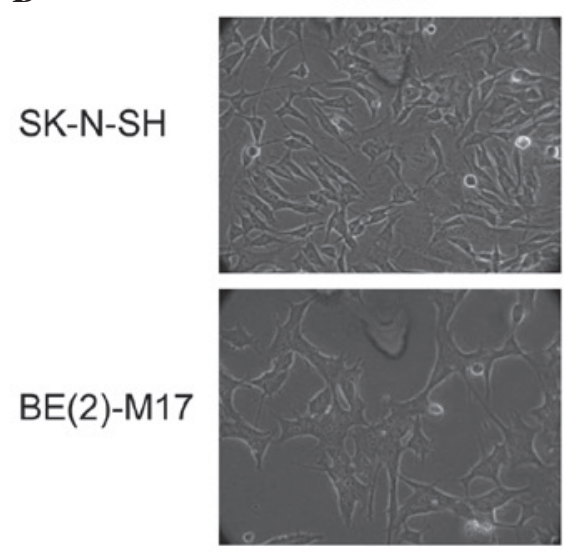

$50 \mu \mathrm{mol} / \mathrm{l}$ Cordycepin
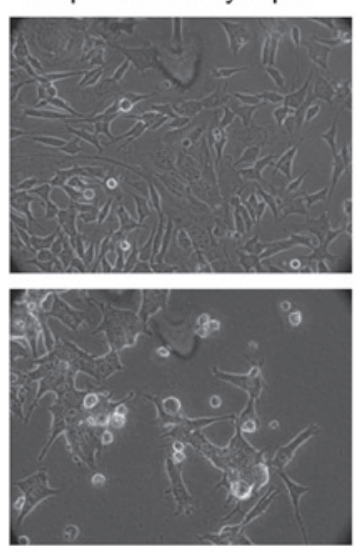

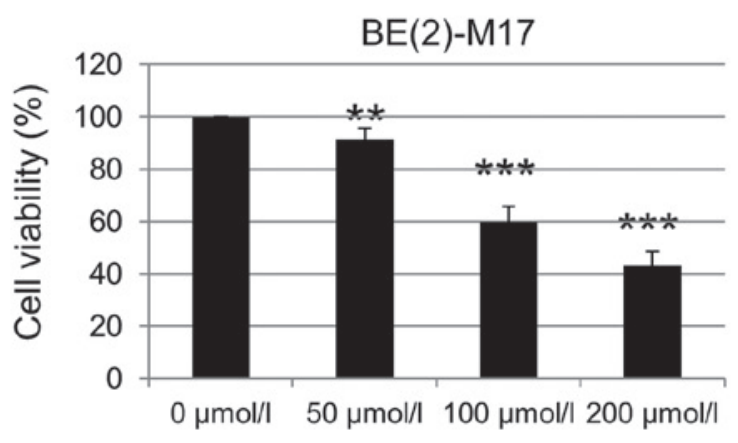

cordycepin

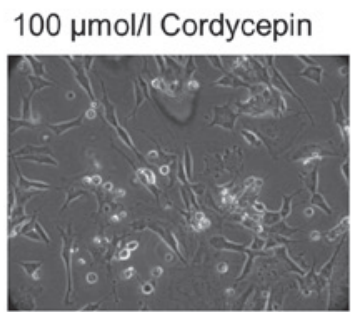

$200 \mu \mathrm{mol} / \mathrm{l}$ Cordycepin
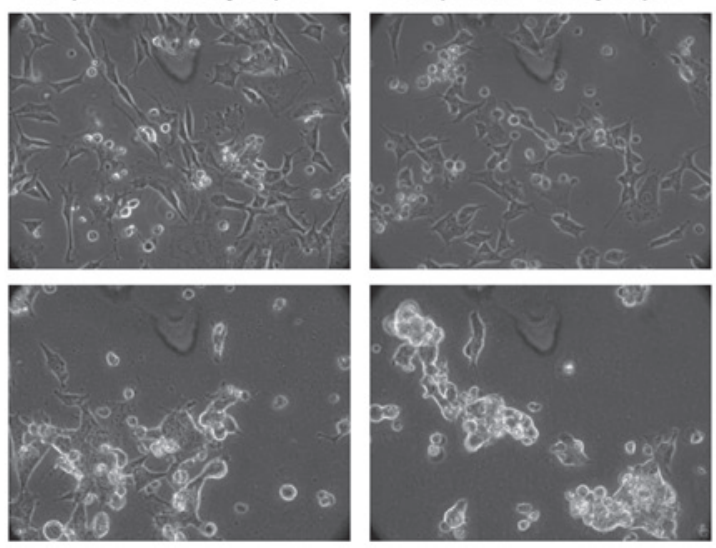

Figure 2. Cordycepin inhibits the viability of SK-N-SH cells. (A) An MTT assay was performed using SK-N-SH and BE(2)-M17 cells treated with cordycepin at the indicated concentrations for $48 \mathrm{~h}$. (B) The morphology of the SK-N-SH and BE(2)-M17 cells treated with the indicated concentrations of cordycepin for $48 \mathrm{~h}$, observed using phase-contrast microscopy (magnification, $\mathrm{x} 40$ ). ${ }^{* * *} \mathrm{P}<0.05$ and ${ }^{* * * *} \mathrm{P}<0.01 \mathrm{vs} .0 \mu \mathrm{mol} / 1$ cordycepin, calculated using the paired Student's $t$-test.

$\mathbf{A}$

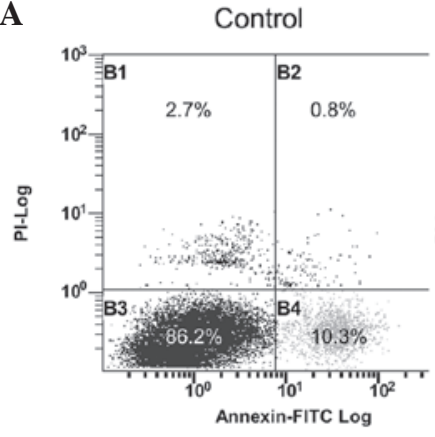

$100 \mu \mathrm{M}$ Cordycepin

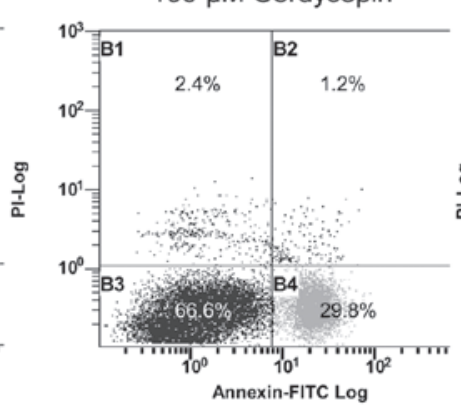

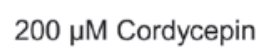

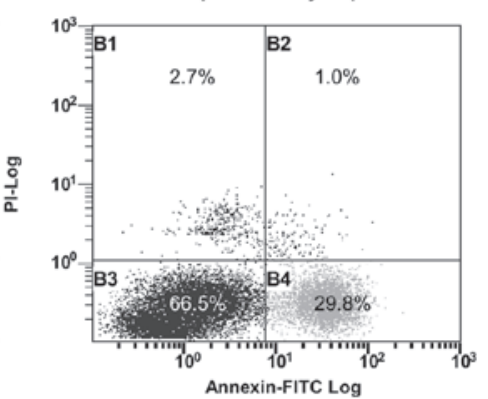

B
Cordycepin ( $\mu \mathrm{mol} / \mathrm{l})$

$\frac{\text { SK-N-SH }}{0 \quad 50100200} \quad \frac{B E(2)-M 17}{050100200}$

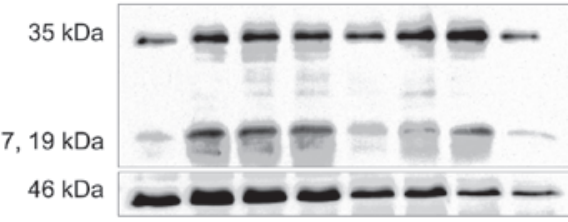

Caspase 3

Cleaved Caspase-3

$\beta$-actin

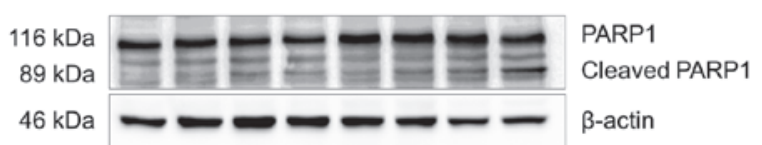

Figure 3. Cordycepin induces apoptotic cell death in neuroblastoma cells. (A) The SK-N-SH cells were treated with 0, 100 and $200 \mu$ mol/l cordycepin for $48 \mathrm{~h}$ prior to staining with Annexin V-PI and assessment using fluorescence-activated cell sorting analysis. (B) Western blot analysis was performed to determine the level of apoptotic markers in SK-N-SH cells treated with cordycepin. $\beta$-actin was used as a loading control. The bands between the full length 116-kDa and cleaved 89-kDa PARP1 bands are non-specific. PI, propidium iodide; FITC, fluorescein isothiocynate; PARP1, poly[ADP-ribose] polymerase 1. 

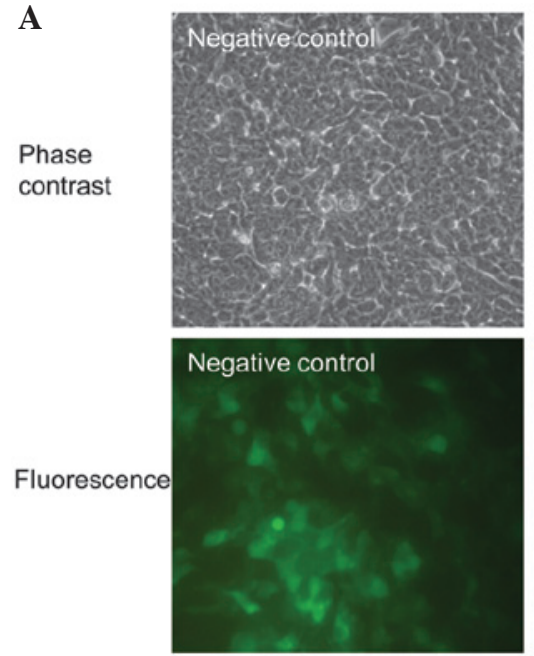

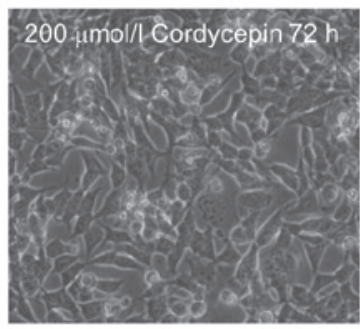

$200 \mu \mathrm{mol} / / \mathrm{l}$ Cordycepin $72 \mathrm{~h}$

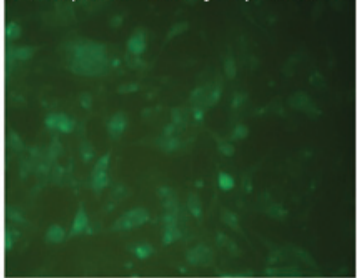

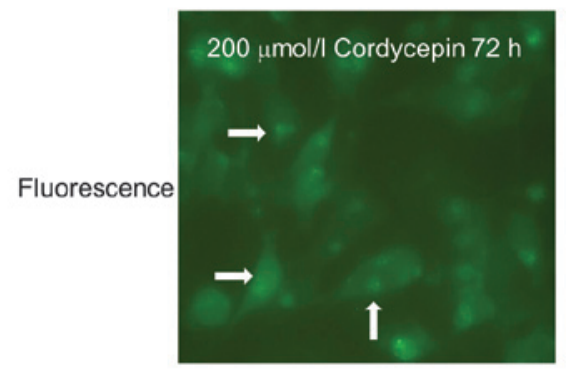

B

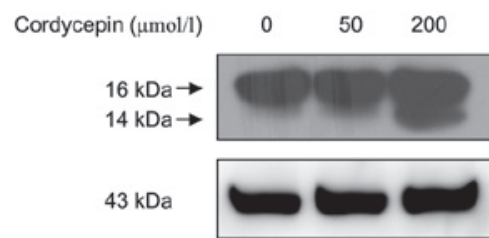

LC3A/B-I

LC3AVB-II

B-actin

Figure 4. Cordycepin induces autophagy in neuroblastoma SK-N-SH cells. (A) SK-N-SH cells stably transfected with the pEGFP-LC3 vector were treated with $200 \mu \mathrm{mol} / 1 \mathrm{l}$ cordycepin for $72 \mathrm{~h}$. The cell morphology and green fluorescence was observed under a microscope. Arrows indicate cells exhibiting a punctate pattern of fluorescence. (B) The effects of cordycepin on LC3 A/B cleavage in SK-N-SH cells was measured by western blot analysis. $\beta$-actin was used as a loading control. LC3, rat microtubule-associated protein 1 light chain 3.
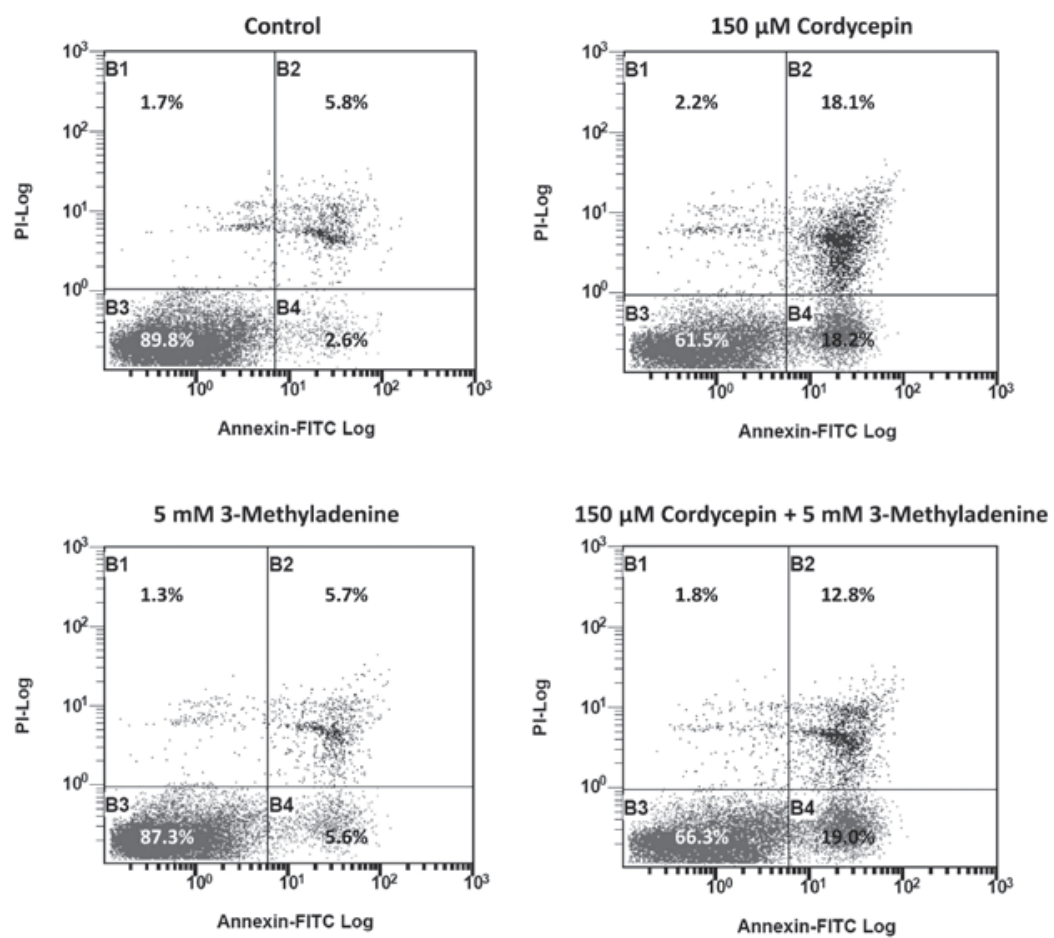

Figure 5. 3-methyladenine (3-Me) alleviates the apoptotic effect of cordycepin on SK-N-SH cells. The SK-N-SH cells were treated with $0 \mu$ mol/1 cordycepin, $150 \mu \mathrm{mol} / 1 \mathrm{cordycepin}, 5 \mathrm{mmol} / \mathrm{l} 3$-methyladenine, or a combination of the latter two, for $48 \mathrm{~h}$ prior to staining with Annexin V-PI and assessment using fuorescence-activated cell sorting analysis.

Viability inhibition of neuroblastoma cells by cordycepin. The effect of the purified cordycepin chemical, one of the bioactive components of Cordyceps sinensis, on the neuroblastoma cells was also investigated. The SK-N-SH cells were treated with $0,50,100$ and $200 \mu \mathrm{mol} / 1$ cordycepin for $48 \mathrm{~h}$, and the cell viability was assessed using an MTT assay. The viability 
of the SK-N-SH and BE(2)-M17 cells was significantly reduced as the cordycepin concentration increased (Fig. 2A). Examination of the SK-N-SH and BE(2)-M17 cells by phase contrast microscopy also revealed an increased number of floating cells and reduced the number of attached cells following treatment with cordycepin, compared with that of non-treated cells (Fig. 2B).

Cordycepin induces apoptosis in BE(2)-M17 and SK-N-SH cells. In order to investigate whether apoptosis occurs in cordycepin-treated neuroblastoma cells, SK-N-SH cells were incubated with or without cordycepin and analyzed by FACS following staining with Annexin V-PI. The percentage of early-apoptotic cells in the SK-N-SH cell populations treated with 100 or $200 \mu \mathrm{mol} / \mathrm{l}$ cordycepin was significantly increased (29.8\%), compared with the percentage of early-apoptotic cells (10.3\%) in the negative control (Fig. 3A). As BE(2)-M17 cells tend to form clusters and do not separate well, apoptosis in this cell line was not assessed by FACS.

The expression of the apoptosis markers caspase- 3 and PARP1 was examined by western blot analysis (29). As shown in Fig. 3B, the expression of the $35 \mathrm{kDa}$ full length caspase-3 protein and its cleavage products were upregulated in the SK-N-SH cells treated with 50, 100 and $200 \mu \mathrm{mol} / \mathrm{l}$ cordycepin, compared with the expression in the non-treated control cells. Similar results were obtained from the $\mathrm{BE}(2)-\mathrm{M} 17$ cells treated with 50 or $100 \mu \mathrm{mol} / 1$ cordycepin. However, in the BE(2)-M17 cells treated with $200 \mu \mathrm{mol} / \mathrm{l}$ cordycepin, caspase-3 upregulation and cleavage was decreased.

In the SK-N-SH and BE(2)-M17 cells, expression of full-length PARP1 was not altered by cordycepin at concentrations up to $200 \mu \mathrm{mol} / 1$. A significant increase in cleavage of PARP1 was observed in SK-N-SH cells treated with 50, 100 or $200 \mu \mathrm{mol} / 1$ cordycepin for $48 \mathrm{~h}$, compared with untreated cells $(\mathrm{P}<0.001)$ (Fig. 3B). In the BE(2)-M17 cells treated with 50,100 or $200 \mu \mathrm{mol} / 1$ cordycepin, PARP1 cleavage increased in a dose-dependent manner, compared with the control samples (Fig. 3B).

Cordycepin induces autophagy in $\mathrm{SK}-\mathrm{N}$-SH cells. The effect of cordycepin treatment on autophagy in the neuroblastoma SK-N-SH cells was assessed using fluorescence microscopy. As shown in Fig. 4A, the SK-N-SH cells that were stably transfected with pEGFP-LC 3 and treated with $200 \mu \mathrm{mol} / 1$ cordycepin for $72 \mathrm{~h}$ exhibited a marked shift from a diffuse to a punctate pattern of LC3-associated green fluorescence (Fig. 4A), indicating that autophagy occurs in cordycepin-treated SK-N-SH cells.

Western blot analysis also revealed increased LC3 A/B protein cleavage in the SK-N-SH cells treated with $200 \mu \mathrm{mol} / \mathrm{l}$ cordycepin compared with the untreated controls (Fig. 4B). However, treatment with $40 \mu \mathrm{mol} / 1$ of cordycepin exhibited no significant effect on autophagy in SK-N-SH cells (Fig. 4B).

3-methyladenine was previously reported to be an autophagy inducer under nutrient-rich conditions (13), which may negatively affect apoptosis. Thus, we investigated the effect of 3-methyladenine on cordycepin-induced apoptosis. The results of FACS revealed that the percentage of late-apoptotic cells in SK-N-SH cell populations treated with $150 \mu \mathrm{mol} / 1$ cordycepin and $5 \mathrm{mM}$ 3 -methyladenine was significantly reduced (12.8\%), compared with the percentage of late-apoptotic cells (18.1\%) in SK-N-SH cells treated with $150 \mu \mathrm{mol} / \mathrm{l}$ cordycepin alone (Fig. 5).

\section{Discussion}

The results of the present study indicate that cordycepin, also termed 3'-deoxyadenosine, induces apoptosis in neuroblastoma cells. Previous studies have demonstrated that polysaccharide-peptide complexes produced by the submerged mycelial culture of an entomopathogenic fungus, Cordyceps sphecocephala, may induce apoptosis in SK-N-SH cells (30). However, the particular components that induced apoptosis remain unclear. To the best of our knowledge, the current study presents the first evidence that the cordycepin chemical alone is able to induce apoptosis in neuroblastoma cells.

Although cordycepin has been reported to induce autophagy in breast cancer cells (19), evidence for cordycepin-induced autophagy in cancer cells remains rare. The present study demonstrated a shift between diffuse and punctate patterns of LC3-associated green fluorescence (Fig. 4A), in addition to an increase in LC3 A/B cleavage (Fig. 4B) in cordycepin-treated neuroblastoma cells compared with the untreated controls. These results indicate that autophagy occurs in neuroblastoma cells treated with cordycepin. It has been reported that Cordyceps militaris and mycelial fermentation induce apoptosis and autophagy in human glioblastoma cells (31). However, whether Cordyceps militaris and mycelial fermentation induce autophagy in neuroblastoma cells remains unclear. In addition, the particular component of Cordyceps militaris and mycelial fermentation that induces autophagy has not been previously reported.

The investigation into the effect of cordycepin on the cleavage of LC3 (Fig. 4B) indicated that low concentrations of cordycepin $(50 \mu \mathrm{mol} / \mathrm{l})$ are able to induce apoptosis but have little effect on autophagy. However, apoptosis and autophagy occurred in SK-N-SH cells treated with cordycepin at a high concentration $(200 \mu \mathrm{mol} / \mathrm{l})$, suggesting that this concentration increases stress in neuroblastoma cells, which respond by initiating autophagy for survival. Previously, 3-methyladenine has been demonstrated to perform a dual role in autophagy; 3-methyladenine has been shown to inhibit starvation-induced autophagy, however, it may also promote autophagic flux following treatment for a prolonged period under nutrient-rich conditions, based on the inhibition of PI3K-III (32). Using FACS analysis, the present study revealed that 3-methyladenine treatment led to a reduction in the proportion of SK-N-SH cells undergoing intermediate-stage cordycepin-induced apoptosis, between 18.1 and $12.8 \%$ (Fig. 5). This observation is consistent with previous studies, which indicated that the inhibition of autophagy triggers apoptosis (33), and therefore reinforces the hypothesis that autophagy is a cytoprotective process under physiological conditions.

The results of the present study showed that cordycepin induces the apoptosis of neuroblastoma SK-N-SH and BE(2)-M17 cells. Furthermore, the study revealed that treatment with cordycepin induces autophagy, as well as apoptosis, in neuroblastoma cells, in a concentration-dependent manner. This suggests that autophagy may counteract apoptosis and serve as a cell survival mechanism. Furthermore, 
3-methyladenine, a promoter of autophagy at full growth medium conditions, inhibited cordycepin induced apoptosis of SK-N-SH cells, which indicated that apoptosis and autophagy are inversely correlated. Future studies, which investigate the inhibition of autophagy following treatment with cordycepin, may improve the therapeutic efficacy of cordycepin neuroblastoma treatment.

\section{Acknowledgements}

The authors would like to thank Professor Cho Chi Hin and Dr Lin Zhang at the Chinese University of Hong Kong for kindly providing the pEGFP-LC3 vector. This study was funded by the National Natural Science Foundation of China (grant no., 31100943), Shenzhen City Science and Technology Project (grant no., 201102136) and Shenzhen Nanshan Science and Technology Research Fund (grant no., 2010012).

\section{References}

1. Gurney JG, Ross JA, Wall DA, Bleyer WA, Severson RK and Robison LL: Infant Cancer in the U.S.: histology-specific incidence and trends, 1973 to 1992. J Pediatr Hematol Oncol 19: 428-432, 1997

2. Spix C,Pastore G, Sankila R, Stiller CA and Steliarova-Foucher E: Neuroblastoma incidence and survival in European children (1978-1997): report from the Automated Childhood Cancer Information System project. Eur J Cancer 42: 2081-2091, 2006.

3. Brodeur GM: Neuroblastoma: biological insights into a clinical enigma. Nat Rev Cancer 3: 203-216, 2003.

4. Maris JM,Hogarty MD, Bagatell R and Cohn SL: Neuroblastoma. Lancet 369: 2106-2120, 2007.

5. Brodeur GM, Pritchard J, Berthold F, et al: Revisions of the international criteria for neuroblastoma diagnosis, staging, and response to treatment. J Clin Oncol 11: 1466-1477, 1993.

6. National Cancer Society: Neuroblastoma risk groups. (http:// www.cancer.org/cancer/neuroblastoma/detailedguide/neuroblastoma-risk-groups). Accessed February 16, 2015.

7. Al-Salem AH (ed): An Illustrated Guide to Pediatric Surgery. Springer International Publishing AG, Switzerland, 2014.

8. D'Angio GJ, Evans AE and Koop CE: Special pattern of widespread neuroblastoma with a favourable prognosis. Lancet 1: 1046-1049, 1971

9. Brodeur GM, Seeger RC, Barrett A, et al: International criteria for diagnosis, staging, and response to treatment in patients with neuroblastoma. J Clin Oncol 6: 1874-1881, 1988.

10. Diede SJ: Spontaneous regression of metastatic cancer: learning from neuroblastoma. Nat Rev Cancer 14: 71-72, 2014.

11. Hallett A and Traunecker H: A review and update on neuroblastoma. Paediatrics and Child Health 22: 103-107, 2012.

12. Cheung NK and Dyer MA: Neuroblastoma: developmental biology, cancer genomics and immunotherapy. Nat Rev Cancer 13: 397-411, 2013.

13. Maris JM: Recent advances in neuroblastoma. N Engl J Med 362: 2202-2211, 2010.

14. Matthay KK, Villablanca JG, Seeger RC, et al: Treatment of high-risk neuroblastoma with intensive chemotherapy, radiotherapy, autologous bone marrow transplantation, and 13-cis-retinoic acid. N Engl J Med 341: 1165-1173, 1999.
15. Kondrashov A, Meijer HA, Barthet-Barateig A, et al: Inhibition of polyadenylation reduces inflammatory gene induction. RNA 18: 2236-2250, 2012.

16. Tuli HS, Sharma AK, Sandhu SS and Kashyap D: Cordycepin: a bioactive metabolite with therapeutic potential. Life Sci 93: 863-869, 2013

17. Siev M, Weinberg R and Penman S: The selective interruption of nucleolar RNA synthesis in HeLa cells by cordycepin. J Cell Biol 41: 510-520, 1969.

18. Hollerer I, Grund K, Hentze MW and Kulozik AE: mRNA 3'end processing: A tale of the tail reaches the clinic. EMBO Mol Med 6: 16-26, 2014.

19. Choi S, Lim MH, Kim KM, Jeon BH, Song WO and Kim TW: Cordycepin-induced apoptosis and autophagy in breast cancer cells are independent of the estrogen receptor. Toxicol Appl Pharmacol 257: 165-173, 2011.

20. Lee SY, Debnath T, Kim SK and Lim BO: Anti-cancer effect and apoptosis induction of cordycepin through DR3 pathway in the human colonic cancer cell HT-29. Food Chem Toxicol 60: 439-447, 2013

21. Chen YH, Hao LJ Hung CP, Chen JW, Leu SF and Huang BM: Apoptotic effect of cisplatin and cordycepin on OC3 human oral cancer cells. Chin J Integr Med 20: 624-632 2014.

22. Jen CY, Lin CY, Huang BM and Leu SF: Cordycepin Induced Ma-10 Mouse Leydig Tumor Cell Apoptosis through Caspase-9 Pathway. Evid Based Complement Alternat Med 2011: 984537, 2011.

23. Wu WC, Hsiao JR, Lian YR, Lin CY and Huang BM: The apoptotic effect of cordycepin on human OEC-M1 oral cancer cell line. Cancer Chemother Pharmacol 60: 103-111, 2007.

24. National Cancer Institute: Chemotherapy with cordycepin plus pentostatin in treating patients with refractory acute lymphocytic or chronic myelogenous leukemia. http://www.cancer.gov/clinicaltrials $/$ search $/$ view $? \mathrm{cdrid}=65572 \&$ version $=$ healthprofessional . Accessed June 10, 2014.

25. Huang TT, Chong KY, Ojcius DM, et al: Hirsutella sinensis mycelium suppresses interleukin-1 $\beta$ and interleukin-18 secretion by inhibiting both canonical and non-canonical inflammasomes. Sci Rep 3: 1374, 2013.

26. Li C, Li Z, Fan M, et al: The composition of Hirsutella sinensis, anamorph of Cordyceps sinensis. J Food Compost Anal 19: 800-805, 2006.

27. Szemes M, Dallosso AR, Melegh Z, et al: Control of epigenetic states by WT1 via regulation of de novo DNA methyltransferase 3A. Hum Mol Genet 22: 74-83, 2013.

28. Kabeya Y, Mizushima N, Ueno T, et al: LC3, a mammalian homologue of yeast Apg8p, is localized in autophagosome membranes after processing. EMBO J 19: 5720-5728, 2000.

29. Elmore S: Apoptosis: a review of programmed cell death. Toxicol Pathol 35: 495-516, 2007.

30. Oh JY, Baek YM, Kim SW, et al: Apoptosis of human hepatocarcinoma (HepG2) and neuroblastoma (SKN-SH) cells induced by polysaccharides-peptide complexes produced by submerged mycelial culture of an entomopathogenic fungus Cordyceps sphecocephala. J Microbiol Biotechnol 18: 512-519, 2008.

31. Yang CH, Kao YH, Huang KS, Wang CY and Lin LW: Cordyceps militaris and mycelial fermentation induced apoptosis and autophagy of human glioblastoma cells. Cell Death Dis 3: e431, 2012

32. Wu YT, Tan HL, Shui G, et al: Dual role of 3-methyladenine in modulation of autophagy via different temporal patterns of inhibition on class I and III phosphoinositide 3-kinase. J Biol Chem 285: 10850-10861, 2010.

33. Boya P, González-Polo RA, Casares N, et al: Inhibition of macroautophagy triggers apoptosis. Mol Cell Biol 25: 1025-1040, 2005. 